\title{
IDM-1, a new zeolite with intersecting medium and extra-large pores built as an expansion of zeolite MFI
}

Luis A. Villaescusa ${ }^{+\dagger}$, Jian $\mathrm{Li}^{+\ddagger}$, Zihao Gao ${ }^{\|}$, Junliang Sun ${ }^{\ddagger \star}$, and Miguel A. Camblor ${ }^{\| \star}$

${ }^{\dagger}$ Instituto Interuniversitario de Investigación de Reconocimiento Molecular y Desarrollo Tecnológico (IDM);

Departamento de Química, Universitat Politècnica de València (UPV); and CIBER de Bioingeniería, Biomateriales y Nanomedicina (CIBER-BBN). Camino de Vera s/n, 46022 Valencia, Spain

${ }^{\ddagger}$ College of Chemistry and Molecular Engineering, Beijing National Laboratory for Molecular Sciences, Peking University,5 Yiheyuan Road, Beijing, 100871, China

${ }^{\perp}$ Berzelii Center EXSELENT on Porous Materials, Department of Materials and Environmental Chemistry, Stockholm University, Stockholm, 10691, Sweden

"Instituto de Ciencia de Materiales de Madrid, Consejo Superior de Investigaciones Científicas (ICMM-CSIC), c/Sor Juana Inés de la Cruz 3, Madrid 28049, Spain

+ : equal author contribution

Correspondence emails: lvillaes@qim.upv.es, junliang.sun@pku.edu.cn, macamblor@icmm.csic.es

\section{Abstract}

IDM-1 is a new silica zeolite with an ordered and well-defined framework constructed by alternating pentasil layers and interrupted layers, giving rise to an intersecting system of straight medium pores and undulating extra-large lobed pores. This unique structure was solved by rotation electron diffraction and refined against synchrotron powder X-ray diffraction data. Despite the presence of both $\mathrm{Si}(\mathrm{OSi})_{3}(\mathrm{OH})$ and $\mathrm{Si}(\mathrm{OSi})_{2}(\mathrm{OH})_{2}$ sites this new zeolite presents high thermal stability, withstanding calcination even to $1000{ }^{\circ} \mathrm{C}$. The location of defects at specific sites of the structure results in alternating hydrophobic $\mathrm{SiO}_{2}$ and hydrophilic $\mathrm{SiO}_{(2-x)}(\mathrm{OH})_{2 x}$ intracrystalline regions. This peculiar combination of intersecting medium and extra-large pores and alternating regions of different chemical character may provide this zeolite with unique catalytic properties. 
Keywords - zeolites, structure solution, rotation electron diffraction, extra-large pores, pentasil

The wide applicability of crystalline microporous materials known as zeolites heavily relies on the wide diversity of their structures and, specifically, of their porosities. The well defined zeolite pores serve as sieves in selective gas separation processes and as nanovessels providing a confined space in which chemical reactions occur with what is known as shape selectivity. $[1,2]$ For this it is understood that the size and shape of the pores may impose restrictions to reactants, transition states and products, thereby determining the selectivity of a particular reaction. In this respect there has been significant interest in the preparation of zeolites with interconnected mixed pores. Traditionally, zeolite pores have been classified as small (8-membered ring, or 8MR, pores, i.e., opened through windows made of $8 \mathrm{TO}_{4}$ tetrahedra, where $\mathrm{T}$ is a tetrahedral framework atom such as $\mathrm{Si}$ or $\mathrm{Al}$ ), medium (10MR), large (12MR) and extra-large pores (>12MR). Nowadays, odd-membered rings are also known, albeit they are scarce, and the classification still holds. The discovery of the natural zeolite boggsite, [3] with an intersecting system of 10 and 12MR pores, prompted the search for interconnected mixed 10/12MR synthetic zeolites, in the believe that they could show interesting shape selectivity properties and applications.[4] Since then, several mixed 10/12MR zeolites have been discovered,[517] while a few silicogermanates with interconnected larger pores have been also synthesized.[1820] However, only very recently a silica zeolite containing medium and extra-large pores has been prepared.[21] High silica zeolites are generally preferred over silicogermanates because of the superior thermal, hydrothermal and chemical stability of the former.[22, 23] Here we present IDM-1, a new silica zeolite with interconnected medium and extra-large pores that is a structural expansion of the well-known zeolite ZSM-5 (MFI zeolite framework type)[24] and presents very high thermal stability. Structural expansion is a formal way of enlarging pores, as in the case of the so-called $\sigma$ expansion, by which all the T-atoms lying in a specific plane are doubled into a T-O-T group.[25] In the case of IDM-1, intercalation of new, complex, multiatom layers between MFI's layers enlarge one of the medium pores of this zeolite into 16MR extra-large pores.

The new zeolite IDM-1 has been synthesized using the organic diquaternary ammonium cation $p$-phenylenedimethylene-bis(tripropylammonium) as a structure-directing agent, at $150{ }^{\circ} \mathrm{C}$, and the details are provided in the Supporting Information. This pure silica material is stable to calcination even at $1000^{\circ} \mathrm{C}$ (Supplementary Fig S1). The calcined material has a type Ia $\mathrm{N}_{2}$ adsorption isotherm, characteristic of microporous materials with narrow micropores $(<1 \mathrm{~nm})($ Fig S2), [26] and it lacks a step typically observed in MFI zeolites around $P / P_{0}=0.15-0.2$, attributed to an adsorbate fluid-like 


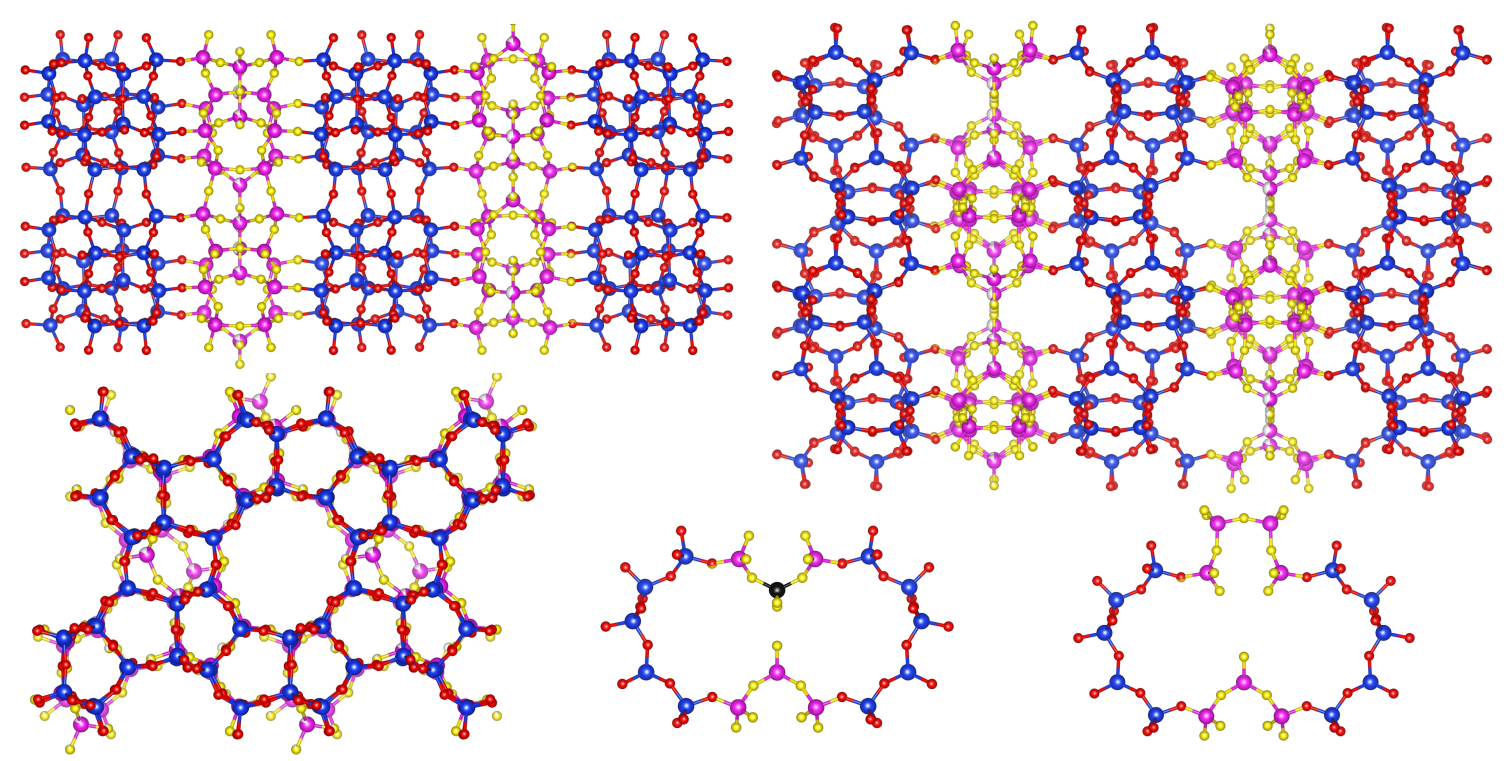

Figure 1: Structure of zeolite IDM-1 along [001] (top right), [100] (top left) and [010] (bottom left). The latter projection, showing the approximately circular 10MR straight pore, is almost identical to that of MFI, except for the $Q^{3}$ site depicted in magenta, which relaxes to a position out of MFI's projection. The IDM-1's characteristic 16MR lobed undulating pore opens through the two-lobed window showed at the bottom center, where a $Q^{2}$ site is shown in black. This pore converts into a $17 \mathrm{MR}$ one (bottom right) when this $Q^{2}$ is missing, thus producing two $Q^{3}$. The pentasil layers topologically identical to MFI's are represented in blue $(\mathrm{Si})$ and red $(\mathrm{O})$, while those belonging to the defective IDM-1's slices are represented in magenta $(\mathrm{Si})$ and yellow $(\mathrm{O})$.

The basic structure of IDM-1 was solved using continuous rotation electron diffraction data, cRED (the original data and reconstructed 3D reciprocal lattice are shown in Movies S1 and S2, respectively). Ab initio structure solution was performed using Superflip,[28] in Pnma symmetry, the obtained structure was anisotropically refined with JANA, [29] and the details are provided in the Supplementary Information. This structure model of calcined IDM-1 is an interrupted framework and there was, however, some ambiguity related to the existence and density of $Q^{3}$ and $Q^{2}$ Si crystallographic sites, i.e., $\mathrm{Si}(\mathrm{OSi})_{3}(\mathrm{OH})$ and $\mathrm{Si}(\mathrm{OSi})_{2}(\mathrm{OH})_{2}$ species, respectively. To solve the ambiguity, the structure model obtained from cRED data was further refined against synchrotron powder X-Ray diffraction data. Rietveld refinement converged to $R_{p}=0.05581, R_{w p}=0.06459$ and $R_{e x p}=0.02154$ with chemically reasonable bond distances and angles (Figure 2, Table S2, TableS3, TableS4). Further 


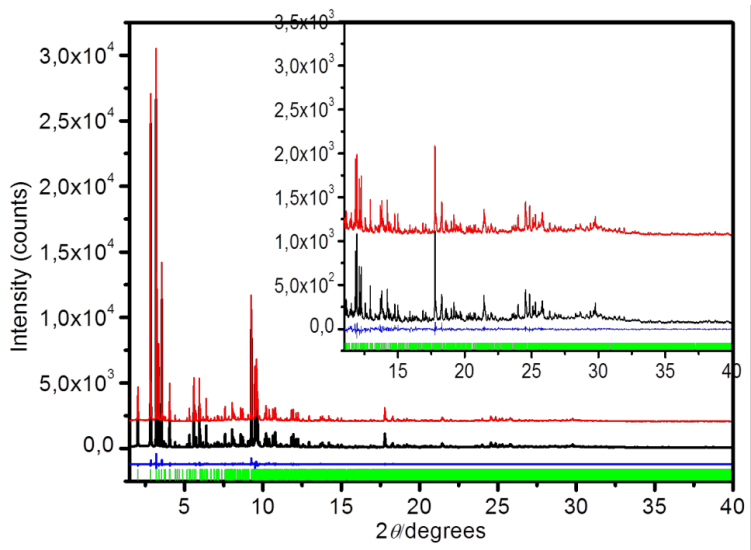

Figure 2: Rietveld refinement plot for Calcined IDM-1 $(\lambda=0.619274 \AA$ ). The curves from top to bottom are simulated, observed, and difference profiles respectively. Inset: scaled up high $2 \theta$ angle. Vertical lines mark allowed reflections.

details are provided in the Supplementary Information. CCDC 1973704 contains the supplementary crystallographic data for this paper. These data are provided free of charge by The Cambridge Crystallographic Data Centre.

To describe the structure of IDM-1 it is convenient to refer first to the classical pentasil unit (Fig. S4): a 14 T-atom unit of eight fused 5MR which can yield pentasil chains by sharing 5MR. Pentasil layers may then be built by connecting layers related by a mirror plane or an inversion center, resulting in what we will call here $m$ - and $i$-pentasil layers, respectively. The typical description of MFI (and MEL) refers to $m$-pentasil layers,[24] but both kinds of pentasil layers actually exist in MFI (MEL only contains $m$-pentasil layers, though). The structure of IDM-1 may be described as consisting of four different but closely related layers or slices normal to [010] (Fig. 1). The first layer is an $i$-pentasil layer as that found in MFI, and is followed by a structurally defective (as opposed to randomly defective) but well-defined slice that may be better described as a collection of unconnected chains rather than as a layer (Fig. S5). This is followed along [010] by a second $i$-pentasil layer (related to the former $i$-pentasil layer by a mirror plane), which in turn is followed by a second incomplete slice related to the previous one by an inversion center. Direct connection of the pentasil layers would yield the MFI topology, i.e., the new structure may be considered an expansion of the MFI zeolite in which successive pentasil layers are separated by defective slices. Interestingly, the defective slices contain incomplete pentasil units, and are thus much related to the pentasil units from which pentasil layers and zeolites are constructed (see Fig. S5 and compare with Fig. 1 bottom left). Here we note the similarity and differences between tetrapropylammonium (TPA), the most typical structure-directing 
agent (SDA) for ZSM-5, and the new SDA reported here, in which a central benzene ring joins two tripropylammonium moieties. As a whole this SDA much resembles two rigidly paired TPA (Figure S6). Further studies regarding structure-direction in this system are in progress.

With regard to connectivity defects and the details of the topology and pore system, the defective slices referred to above contain $Q^{3}$ (sites 21 and 22, 4 each per unit cell) and $Q^{2}$ sites (Si17, $4 / \mathrm{uc}$ ). However, by ${ }^{29} \mathrm{Si}$ MAS NMR (Fig. S7) and Rietveld refinement we found that these sites, and also Si20 (connecting Si21 and Si22) have partial occupancies, implying they are occasionally missing. This affects the pore size of one of the two types of pores that IDM-1 contains. The first one is a straight, basically circular 10MR pore running along the [010] direction and it intersects the second one, an undulating pore with elongated windows that runs approximately along [100]. When the $Q^{2}$ is present the second pore can be described as a lobed, peanut-shaped, 16MR pore (Figure 1, bottom center). When these $Q^{2}$ sites are missing, with $40 \%$ probability according to Rietveld refinement, the window is a $17 \mathrm{MR}$ asymmetrical pore (Figure 1, bottom right).

In conclusion, IDM-1 is a new microporous silica polymorph which represents a structural expansion of the classical zeolite ZSM-5 and contains a system of interconnected medium and extra-large pores. Despite the defective nature of its framework, this zeolite shows a remarkably high thermal stability as it is able to withstand heating temperatures as high as $1000{ }^{\circ} \mathrm{C}$ without collapsing. Additionally, $\mathrm{Al}$ can be introduced in the framework, and this may likely be possible for other catalytically active heteroatoms, such as Ti. In our opinion, the unique pore system of IDM-1 and the presence of alternating regularly spaced hydrophobic (all Q4) and hydrophilic regions (containing $Q^{3}$ and $Q^{2}$ sites) may provide interesting opportunities for further developments. For instance, catalytic reactions involving hydrophilic and hydrophobic reactants may benefit from the presence of hydrophobic and hydrophilic environments.[30] Finally, active moieties could be bonded to $Q^{2}$ and $Q^{3}$ sites giving the material a further functionality.

\section{Acknowledgement}

The authors thank funding from the Spanish Ministry of Science, Innovation and Universities, under Projects MAT2015-71117-R (MINECO/FEDER, UE) and RTI2018-101599-B-C22 (MCUI/AEI/FEDER, UE), the National Natural Science Foundation of China (No. 21871009, 21621061, 21527803, 21471009), the Swedish Research Council (VR) and the Knut and Alice Wallenberg Foundation (KAW). L.A.V. also thanks the Generalitat Valenciana (project PROMETEO/2018/024). Synchrotron experiments were performed at beamline BL04 (MSPD) at ALBA Synchrotron with the collaboration of ALBA staff, 
and especial thanks are due to A. Manjón for support in collecting the data and for helpful comments and suggestions.

\section{Conflicts of interest}

L.A.V. has filed a patent application on zeolite IDM-1.

\section{References}

[1] P. B. Weisz, Pure Appl. Chem. 1980, 52, 2091-2103.

[2] S. M. Csicsery, Zeolites 1984, 4, 202-213.

[3] J. J. Pluth, J. V. Smith, Am. Mineral. 1990, 75, 501-507.

[4] R. F. Lobo, M. Pan, I. Chan, H.-X. Li, R. C. Medrud, S. I. Zones, P. A. Crozier, M. E. Davis, Science 1993, 262, 1543-1546.

[5] P. A. Wright, R. H. Jones, S. Natarajan, R. G. Bell, J. Chen, M. B. Hursthouse, J. M. Thomas, J. Chem. Soc., Chem. Commun. 1993, 633-635.

[6] L. Josien, A. Simon-Masseron, V. Gramlich, J. Patarin, L. Rouleau, Chemistry - A European Journal 2003, 9, 856-861.

[7] R. Castañeda, A. Corma, V. Fornés, F. Rey, J. Rius, J. Am. Chem. Soc. 2003, 125, 7820-7821.

[8] D. L. Dorset, S. C. Weston, S. S. Dhingra, J. Phys. Chem. B 2006, 110, 2045-2050.

[9] S. Elomari, A. Burton, R. C. Medrud, R. Grosse-Kunstleve, Microporous and Mesoporous Materials 2009, 118, 325-333.

[10] M. Dodin, J.-L. Paillaud, Y. Lorgouilloux, P. Caullet, E. Elkaïm, N. Bats, J. Am. Chem. Soc. 2010, 132, 10221-10223.

[11] D. Xie, L. B. McCusker, C. Baerlocher, J. Am. Chem. Soc. 2011, 133, 20604-20610.

[12] C. Baerlocher, T. Weber, L. B. McCusker, L. Palatinus, S. I. Zones, Science 2011, 333, 11341137.

[13] M. Moliner, T. Willhammar, W. Wan, J. González, F. Rey, J. L. Jorda, X. Zou, A. Corma, J. Am. Chem. Soc. 2012, 134, 6473-6478.

[14] T. Willhammar, J. Sun, W. Wan, P. Oleynikov, D. Zhang, X. Zou, M. Moliner, J. Gonzalez, C. Martínez, F. Rey, A. Corma, Nature Chemistry 2012, 4, 188-194. 
[15] E. Verheyen, L. Joos, K. Van Havenbergh, E. Breynaert, N. Kasian, E. Gobechiya, K. Houthoofd, C. Martineau, M. Hinterstein, F. Taulelle, V. Van Speybroeck, M. Waroquier, S. Bals, G. Van Tendeloo, C. E. A. Kirschhock, J. A. Martens, Nature Materials 2012, 11, 1059.

[16] Y. Lorgouilloux, M. Dodin, E. Mugnaioli, C. Marichal, P. Caullet, N. Bats, U. Kolb, J.-L. Paillaud, RSC Adv. 2014, 4, 19440-19449.

[17] S. A. Morris, G. P. M. Bignami, Y. Tian, M. Navarro, D. S. Firth, J. Cejka, P. S. Wheatley, D. M. Dawson, W. A. Slawinski, D. S. Wragg, R. E. Morris, S. E. Ashbrook, Nat. Chem. 2017, 9, 1012-1018.

[18] J.-L. Paillaud, B. Harbuzaru, J. Patarin, N. Bats, Science 2004, 304, 990-992.

[19] A. Corma, M. J. Diaz-Cabanas, J. L. Jorda, C. Martinez, M. Moliner, Nature 2006, 443, 842845 .

[20] J. H. Kang, D. Xie, S. I. Zones, S. Smeets, L. B. McCusker, M. E. Davis, Chem. Mater. 2016, 28, 6250-6259.

[21] T. Willhammar, A. W. Burton, Y. Yun, J. Sun, M. Afeworki, K. G. Strohmaier, H. Vroman, X. Zou, J. Am. Chem. Soc. 2014, 136, 13570-13573.

[22] S. Valencia, PhD thesis, Universidad Politécnica de Valencia, 1997.

[23] L. A. Villaescusa, M. A. Camblor, Chem. Mater. 2016, 28, 7544-7550.

[24] C. Baerlocher, L. B. McCusker, Database of Zeolite Structures, accessed on October 28th, 2019, http://www.iza-structure.org/databases/.

[25] R. M. Barrer, Hydrothermal Chemistry of Zeolites. 1982.

[26] M. Thommes, K. K., N. A.V., J. Olivier, F. Rodriguez-Reinoso, J. Rouquerol, K. S. W. Sing, Physisorption of gases, with special reference to the evaluation of surface area and pore size distribution (IUPAC Technical Report), 2015.

[27] U. Müller, H. Reichert, E. Robens, K. K. Unger, Y. Grillet, F. Rouquerol, J. Rouquerol, D. Pan, A. Mersmann, Fresenius' Zeitschrift für analytische Chemie 1989, 333, 433-436.

[28] L. Palatinus, G. Chapuis, J. Appl. Cryst. 2007, 40, 786-790.

[29] V. Petricek, M. Dusek, L. Palatinus, Z. Kristallogr. 2014, 229, 345-352.

[30] M. A. Camblor, A. Corma, S. Iborra, S. Miquel, J. Primo, S. Valencia, J. Catal. 1997, 172, $76-84$. 
[31] S. Smeets, B. Wang, M. Cichocka, J. Angstrom, W. Wan, Instamatic (Version 0.6) Zenodo, 2018.

[32] W. Wan, J. Sun, J. Su, S. Hovmöller, X. Zou, J. Appl. Crystallogr. 2013, 46, 1863-1873.

[33] W. Kabsch, Acta Cryst. 2010, D66, 125-132.

[34] A. Coelho, TOPAS-ACADEMIC v.5.0, 2012.

[35] C. Baerlocher, A. Hepp, W. M. Meier, DLS-76. A program for the simulation of crystal structures by geometric refinement, 1976 . 


\section{Supplementary Material}

\section{Synthesis details}

For the preparation of the SDA, $5.00 \mathrm{~g}$ of 1,4-bis(chloromethyl)benzene (Aldrich) (0.02856 mole) was added to a round bottom flask containing $300 \mathrm{~mL}$ of ethanol. Once dissolved, the solution was placed in an ice bath and $24.5 \mathrm{~g}$ of tripropylamine (0.34406 mole)were added dropwise. After 4 days of refluxing, ethanol and some tripropylamine was stripped in a rotavapor until a paste was obtained, which was washed with acetone and dried. The white solid obtained (13.10 grams, 99 \%yield) was recognized by ${ }^{1} \mathrm{H}$ NMR as the chloride salt of the dication $p$-phenylenedimethylene-bis(tripropylammonium).

The salt was anion exchanged using Dowex Monosphere 550A hydroxyde form (Sigma-Aldrich) to yield a solution which was $8 \times 10^{-4}$ mols $\mathrm{OH}$ per gram of solution, as determined by titration.

Zeolite IDM-1 was prepared by adding $3.47 \mathrm{~g}$ of TEOS in a teflon vessel containing 10.40 grams of the previously synthesized $\mathrm{OH}$ solution. The mixture was left under stirring at room temperature until the mass of gel reached 6.94 grams. Then, $0,35 \mathrm{~g}$ of $\mathrm{HF}(48 \% \mathrm{w} / \mathrm{w})$ were added and the produced paste stirred by hand until a homogeneous mixture was achieved. The gel composition was $\mathrm{SiO}_{2}$ : $0.25 \mathrm{RF}_{2}: 0.5 \mathrm{HF}: 14.5 \mathrm{H}_{2} \mathrm{O}$, where $\mathrm{R}$ is the dication. The Teflon vessel was then put into a Parr reactor, sealed, and placed into an oven at $150{ }^{\circ} \mathrm{C}$. After 38 days the reactor was taken out, quenched and the solid recovered by filtration. The mass of the obtained as-made zeolite was $0.55 \mathrm{~g}$. Finally, calcination of the solid at $550{ }^{\circ} \mathrm{C}$ for 5 hours produced the porous solid.

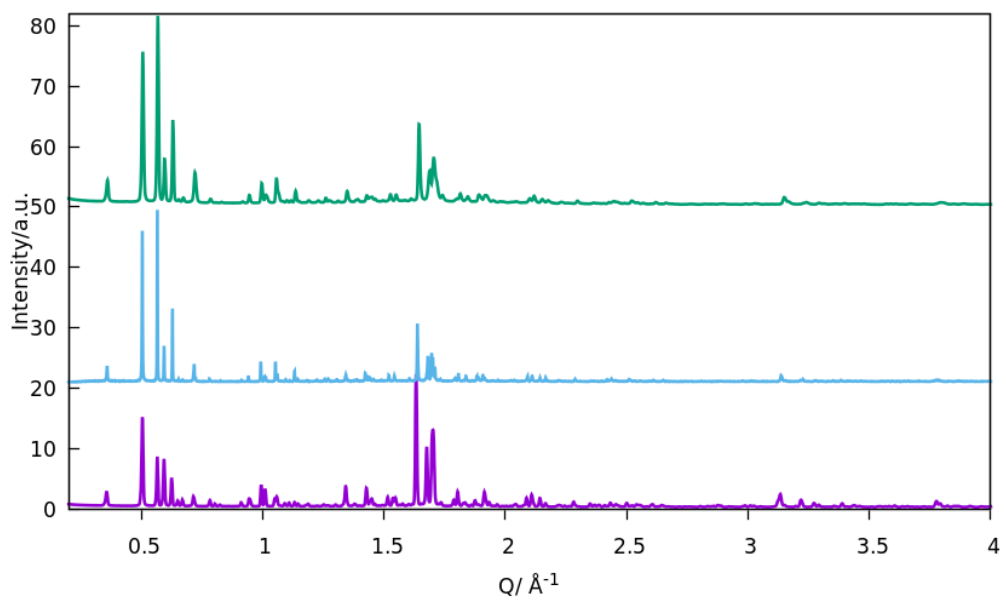

Figure S1: XRD patterns of IDM-1: as-made (bottom) and calcined at 550 (middle) and $1000{ }^{\circ} \mathrm{C}$. 


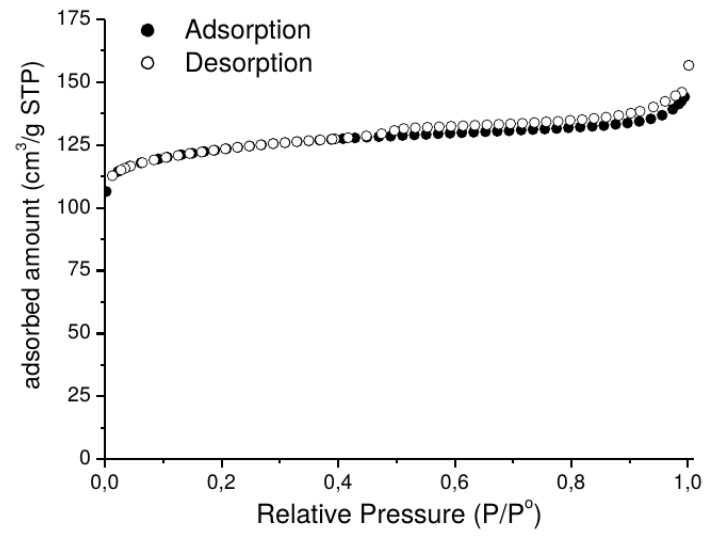

Figure S2: N2 adsorption isotherm of IDM-1 calcined at $550 \circ \mathrm{C}$. 


\section{Structure Solution}

Because of the small crystal size of calcined IDM-1, a 3D electron diffraction technique, continuous rotation electron diffraction (cRED), was applied for structure determination. A cRED dataset was collected on a plate-like crystal. The sample powder was crushed in an agate mortar, dispersed in absolute ethanol and treated by ultrasonication for 5 minutes. Then, a droplet of suspension was transferred to a copper grid. The 3D ED data were collected on 200kV JEOL JEM-2100 transmission electron microscope using the software instamatic.[31] During the data collection, the goniometer was rotated continuously while the selected area ED patterns were captured from the crystal simultaneously by a quad hybrid pixel detector (Timepix). $463 \mathrm{ED}$ patterns were recorded with tilt step of $0.23^{\circ}$, and the tilt range from $-61.10^{\circ}$ to $57.90^{\circ}$.

The 3D reciprocal lattice was reconstructed by the software REDp (Figure S3),[32] which was very useful for indexing and obtaining the reflection conditions(Table S1). This reciprocal lattice shows clearly an mmm Laue symmetry with an orthorhombic symmetry with unit cell parameters a $\approx 20.24 \AA, \mathrm{b} \approx 35.25 \AA$ and $\mathrm{c} \approx 13.51 \AA$. The reflection condition derived from $2 \mathrm{D}$ slice cut from the $3 \mathrm{D}$ reciprocal lattice indicated that the possible space groups of IDM-1 was Pnma (No. 62) or Pn21a (No.33). Data processing was conducted using the software package XDS,[33] which generated the hkl files that were used for structure solution and refinement. Ab inito structure solution was performed with each of the two space groups using Superflip[28] and atomic scattering factors for electrons. 21 crystallographic independent $\mathrm{Si}$ and part of oxygen atoms were located directly within Pnma symmetry, and the remaining oxygen atoms and one $\mathrm{Si}$ atoms were then found from residue peaks. The framework with $22 \mathrm{Si}$ atoms and $45 \mathrm{O}$ atoms in the asymmetric unit could be refined anisotropically without any restraints by JANA 2006.[29]

Synchrotron data were collected in Debye-Scherrer geometry at beamline MSPD of the Spanish synchrotron, ALBA, with a wavelength of $\lambda=0.619274 \AA$. The Rietveld refinement of the calcined IDM-1 was performed with Topas 4.1.[34] Initially, a Pawley fit was performed to optimize the unit cell dimensions, background, and peak profile. The background was modeled with a 14-term shifted Chebyshev polynomial, and the peak profile was modeled with the Pearson VII peak-shape function. Data were used to a d-spacing of $0.9025 \AA$ for a total of 6996 reflections. The model from cRED data was used as initial model. Before the refinement, an optimization of the framework geometry was performed by using the distance-least-squares algorithm in the program DLS-76.[35] In the initial stages of the refinement, soft restraints were placed on the Si-O bond distances $(1.61 \AA)$, the Si-O-Si bond angels $\left(145^{\circ}\right)$ and $\mathrm{O}-\mathrm{Si}-\mathrm{O}$ bond angels $\left(109^{\circ}\right)$. These restraints were gradually reduced and eventually 
Table S1: cRED: Experimental parameters and Crystallographic data of calcined IDM-1.

\begin{tabular}{ll} 
Identification code & IDM-1 \\
Tilt range & $-61.10^{\circ} \sim 57.90^{\circ}$ \\
Tilt step & $0.23^{\circ}$ \\
Wavelength & $0.0251 \AA$ \\
No. of frames & 463 \\
Program for data procession & XDS \\
Program for structure determination & ShelxT/Superflip \\
Crystal system & Orthorhombic \\
Unit cell dimensions & $\mathrm{a}=20.2450 \AA$ \\
& $\mathrm{b}=35.2570 \AA$ \\
Possible space group & $\mathrm{c}=13.5100 \AA$ \\
Resolution & Pnma $/ \mathrm{Pn} 21 \mathrm{a}$ \\
Completeness & $0.82 \AA$ \\
R1 & $77.7 \%$ \\
No. of unique reflections & 0.494 \\
\hline
\end{tabular}

completely relaxed. The four water molecules were located by electron density difference map. After the initial refinement of the framework and water molecules, the occupancies of all the Si atoms were opened to refinement, but only the occupancy values of Si17, Si20, Si21 and Si22 were observed to decrease. Then, the occupancies of Si atoms were fixed except Si17, Si20, Si21 and Si22, and an excellent agreement between the experimental data and simulated data was obtained. The occupancy of Si17, Si20, Si21 and Si22 (together with their dangling O atoms) converged to 0.59268(647), 0.82327(895), $0.84896(888)$ and $0.88875(708)$ respectively. Finally, the unit cell dimensions, background, and peak profile were further refined. Final crystallographic data are summarized in Table S2 and selected distances and angles are presented in Tables S3 and S4, respectively. 


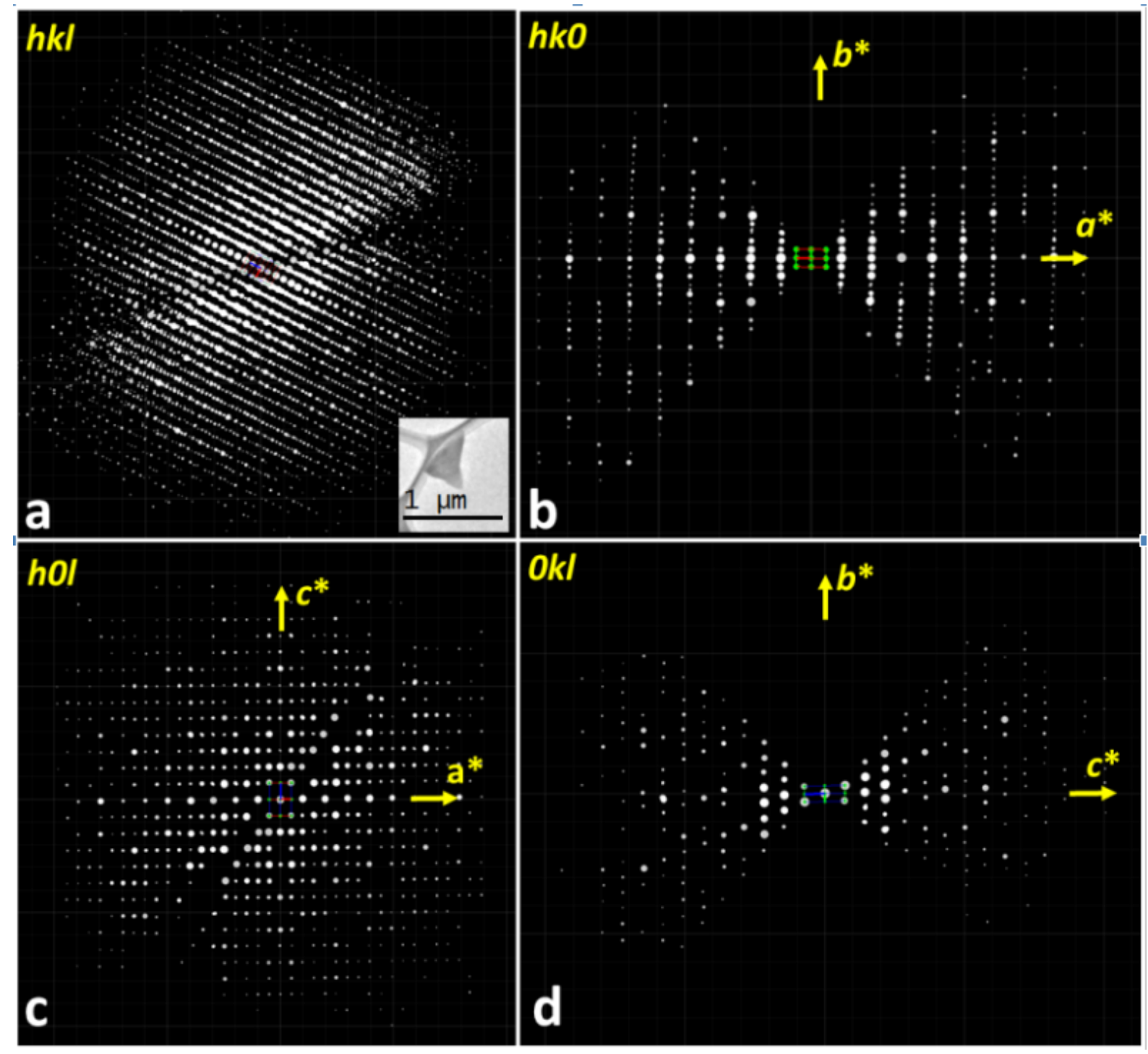

Figure S3: Reconstructed three-dimensional cRED data. a) Overview of the data, b), c), d) selected planes in the reciprocal lattice corresponding to hk0, h0l, and 0kl planes, respectively. Reflection conditions: $0 \mathrm{kl}, \mathrm{k}+\mathrm{l}=2 \mathrm{n} ; \mathrm{hk} 0, \mathrm{~h}=2 \mathrm{n} ; \mathrm{h} 00, \mathrm{~h}=2 \mathrm{n} ; 0 \mathrm{k} 0, \mathrm{k}=2 \mathrm{n} ; 00 \mathrm{l}, \mathrm{l}=2 \mathrm{n}$. 
Table S2: Crystal data and structure refinement for calcined IDM-1

\begin{tabular}{ll} 
Identification code & IDM-1 \\
Empirical formula & {$\left[\mathrm{Si}_{156.6} \mathrm{O}_{322.5} \mathrm{H}_{18.6}\right]\left(\mathrm{H}_{2} \mathrm{O}\right)_{32}$} \\
Wavelength & $0.61927399 \AA$ \\
Radiation & Synchrotron Radiation \\
Crystal system & Orthorhombic \\
Space group & Pnma \\
Unit cell dimensions & $\mathrm{a}=20.03454(23) \AA$ \\
& $\mathrm{b}=35.07043(42) \AA$ \\
& $\mathrm{c}=13.36596(17) \AA$ \\
Volume & $9391.19(20)$ \\
Z & 1 \\
2 theta range for data refinement & $1.5^{\circ}<2 \theta<40^{\circ}$ \\
Number of parameters & 214 \\
Number of reflections & 6996 \\
Number of data points & 12834 \\
Number of restrains & 88 for Si-O and 173 for O-Si-O \\
Refinement method & Rietveld refinement \\
$R p / R w p /$ Rexp & $0.05581 / 0.06459 / 0.02154$ \\
\hline &
\end{tabular}


Table S3: Si-O bond distances $(\stackrel{\circ}{A})$ for calcined IDM-1

\begin{tabular}{|c|c|c|c|c|c|}
\hline Si1-O12 & $1.60291(44)$ & $\mathrm{Si9-O} 24$ & $1.59752(42)$ & Si17-O44 & $1.60820(64)$ \\
\hline Si1-O32 & $1.60411(45)$ & Si9-O9 & $1.60315(47)$ & Si17-O11 & $1.60678(31)$ \\
\hline Si1-O14 & $1.60656(46)$ & $\mathrm{Si9} 9-\mathrm{O} 6$ & $1.61433(56)$ & Si17-O11 & $1.60678(31)$ \\
\hline Si1-O17 & $1.60875(48)$ & $\mathrm{Si9}-\mathrm{O} 7$ & $1.61326(46)$ & Si17-O45 & $1.60802(64)$ \\
\hline Si2-O32 & $1.60699(48)$ & Si10-O19 & $1.60610(50)$ & Si18-O25 & $1.59257(42)$ \\
\hline Si2-O15 & $1.60262(47)$ & Si10-O22 & $1.60540(45)$ & Si18-O23 & $1.60526(44)$ \\
\hline $\mathrm{Si} 2-\mathrm{O} 20$ & $1.61280(46)$ & Si10-O10 & $1.60460(47)$ & Si18-O18 & $1.60735(48)$ \\
\hline Si2-O30 & $1.61208(38)$ & Si10-O13 & $1.60460(47)$ & Si18-O35 & $1.60473(53)$ \\
\hline $\mathrm{Si} 3-\mathrm{O} 25$ & $1.59492(42)$ & Si11-O38 & $1.60114(31)$ & Si19-O24 & $1.59809(47)$ \\
\hline $\mathrm{Si3}-\mathrm{O} 20$ & $1.61200(51)$ & Si11-O2 & $1.60905(51)$ & Si19-O15 & $1.60190(47)$ \\
\hline Si3-O13 & $1.60682(51)$ & Si11-O6 & $1.61354(47)$ & Si19-O5 & $1.60808(45)$ \\
\hline Si3-O17 & $1.61023(49)$ & Si11-O8 & $1.61788(55)$ & Si19-O18 & $1.60800(50)$ \\
\hline $\mathrm{Si} 4-\mathrm{O} 28$ & $1.60086(42)$ & Si12-O28 & $1.59936(42)$ & Si20-O34 & $1.60090(36)$ \\
\hline Si4-O16 & $1.60755(47)$ & Si12-O26 & $1.60105(46)$ & Si20-O34 & $1.60090(36)$ \\
\hline Si4-O22 & $1.60627(51)$ & Si12-O8 & $1.61693(41)$ & Si20-O41 & $1.60902(47)$ \\
\hline Si4-O21 & $1.61253(44)$ & Si12-O36 & $1.61079(50)$ & Si20-O39 & $1.60554(49)$ \\
\hline Si5-O19 & $1.60413(46)$ & Si13-O4 & $1.59797(42)$ & Si21-O26 & $1.60629(36)$ \\
\hline $\mathrm{Si5-O1}$ & $1.60471(50)$ & Si13-O10 & $1.60675(45)$ & Si21-O26 & $1.60629(36)$ \\
\hline $\mathrm{Si} 5-\mathrm{O} 27$ & $1.60835(43)$ & Si13-O14 & $1.61118(53)$ & Si21-O42 & $1.61045(54)$ \\
\hline Si5-O30 & $1.61157(39)$ & Si13-O27 & $1.61147(54)$ & Si21-O41 & $1.61178(44)$ \\
\hline Si6-O37 & $1.59516(42)$ & Si14-O4 & $1.59374(41)$ & Si22-O9 & $1.60516(35)$ \\
\hline Si6-O35 & $1.60525(47)$ & Si14-O16 & $1.60583(45)$ & Si22-O9 & $1.60516(35)$ \\
\hline Si6-O1 & $1.60402(47)$ & Si14-O5 & $1.61047(57)$ & $\mathrm{Si} 22-\mathrm{O} 43$ & $1.61153(61)$ \\
\hline Si6-O31 & $1.61107(59)$ & Si14-O31 & $1.60487(42)$ & Si22-O39 & $1.61080(47)$ \\
\hline Si7-O29 & $1.60479(48)$ & Si15-O29 & $1.60445(50)$ & & \\
\hline Si7-O23 & $1.60569(44)$ & Si15-O33 & $1.60586(57)$ & & \\
\hline Si7-O12 & $1.60195(52)$ & Si15-O36 & $1.61474(41)$ & & \\
\hline Si7-O21 & $1.60947(44)$ & Si15-O11 & $1.60853(53)$ & & \\
\hline Si8-O37 & $1.59106(45)$ & Si16-O40 & $1.60134(38)$ & & \\
\hline Si8-O34 & $1.59639(42)$ & Si16-O33 & $1.60707(39)$ & & \\
\hline Si8-O3 & $1.60781(47)$ & Si16-O3 & $1.61257(51)$ & & \\
\hline Si8-O2 & $1.60831(53)$ & Si16-O7 & $1.61202(56)$ & & \\
\hline
\end{tabular}


Table S4: Bond angels for calcined IDM-1

\begin{tabular}{lll}
\hline Bond Angels $\left(^{\circ}\right)$ & Min & Max \\
\hline Si-O-Si & $134.21(29)$ & $176.42(49)$ \\
O-Si-O & $97.79(29)$ & $125.79(59)$ \\
\hline
\end{tabular}
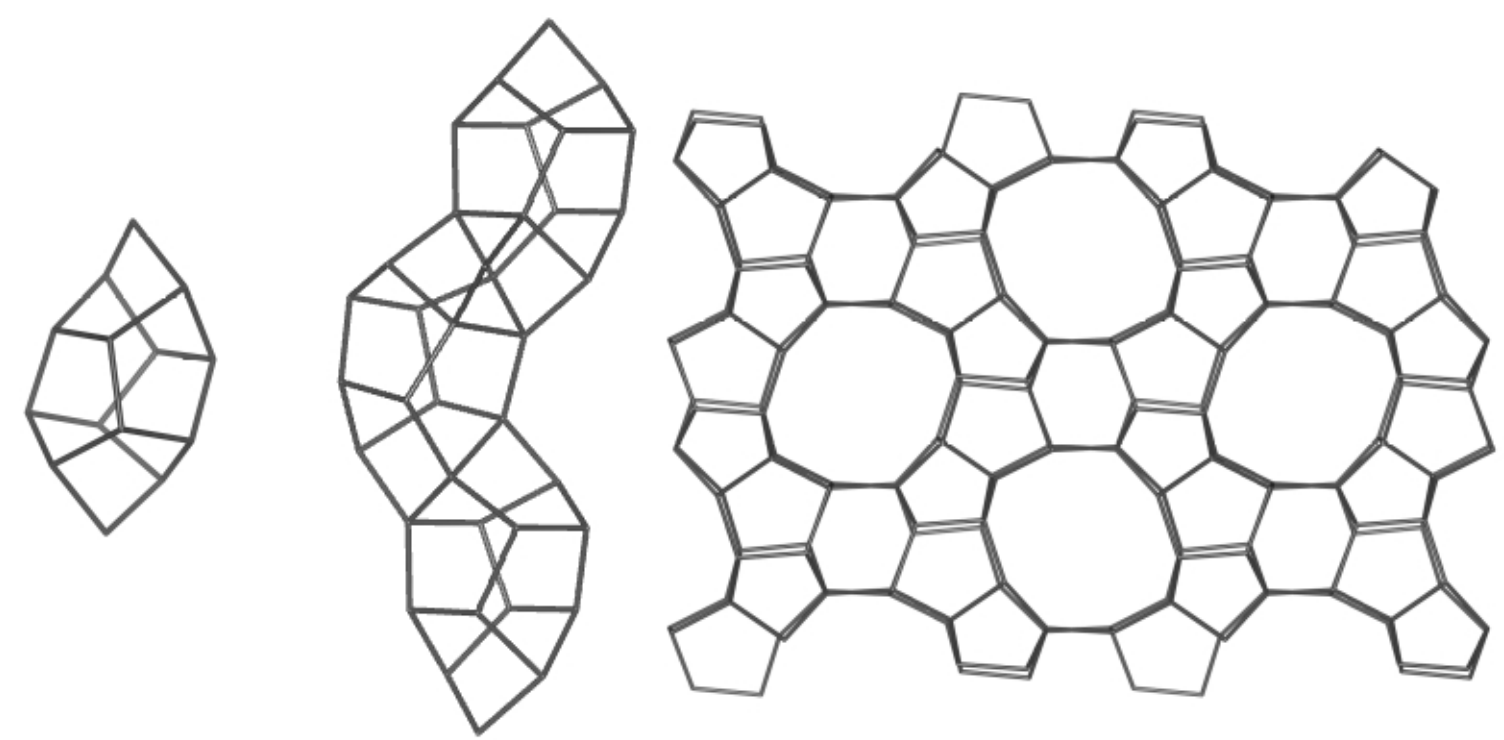

Figure S4: The 14 T-atom pentasil unit (left), the pentasil chain (middle) and the $i$-pentasil layer made by connecting pentasil layers related by and inversion center. Only T-T connectivity is represented. 


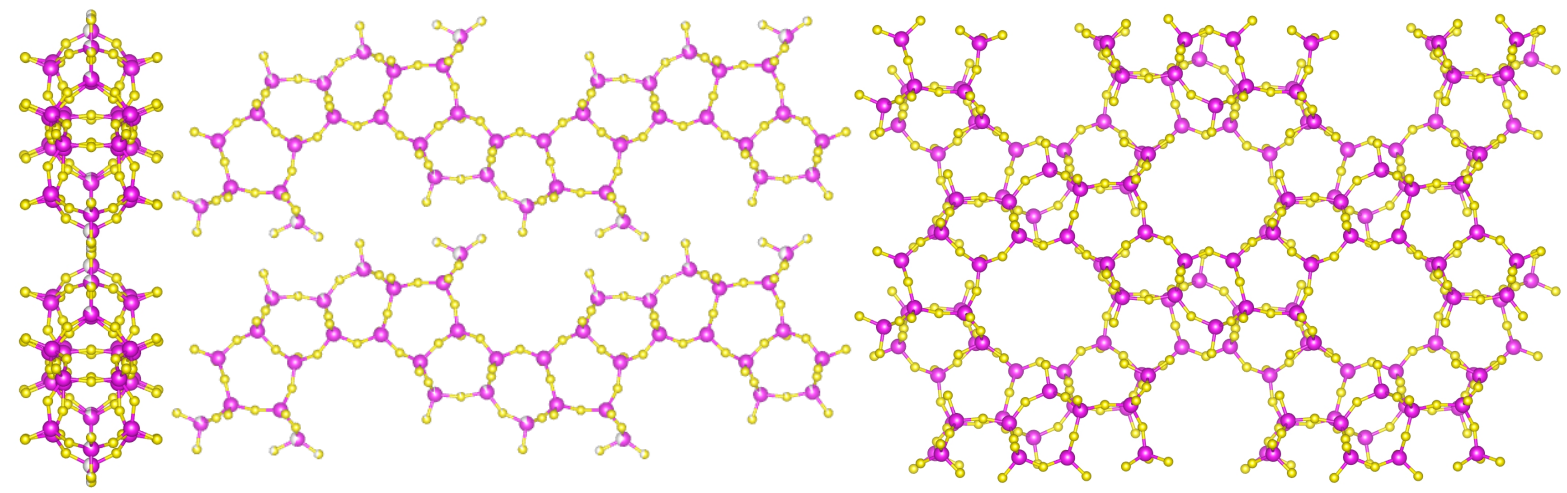

Figure S5: The defective slices between pentasil layers in IDM-1, represented along [100] (left) and [010] (middle, evidencing the chain-like nature and incomplete connectivity of the slice). The projection of two such slices along [010] (right) looks like the projection of the whole IDM-1 structure (Fig. 1, bottom left) and much like a complete MFI pentasil layer, except for a $Q^{3}$ site displaced towards the central region of an apparent $6 \mathrm{MR}$.
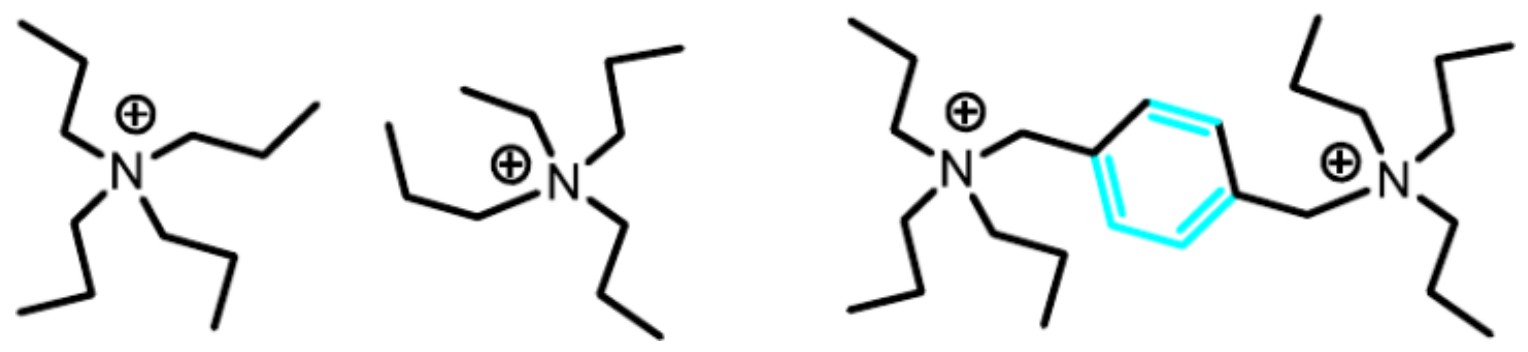

Figure S6: The SDA used to synthesize IDM-1 (right) closely resembles two TPA cations rigidly joined through a benzene ring. 


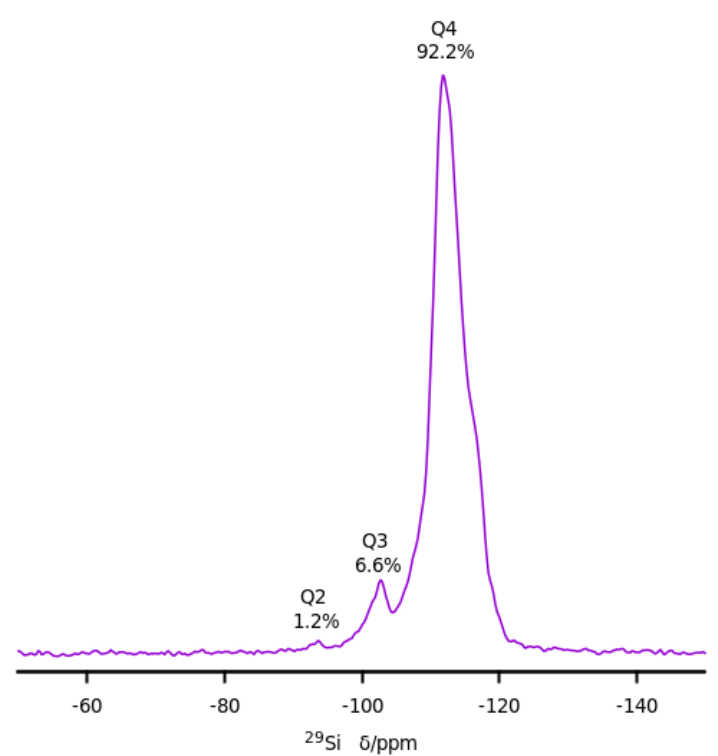

Figure S7: 29Si MAS NMR spectrum of zeolite IDM-1 calcined at $550{ }^{\circ} \mathrm{C}$. 